\title{
Frequency and Dependence of Long Range Temporal Correlations in Human Hippocampal Energy Fluctuations
}

\author{
M. STEAD, ${ }^{1}$ G. A. WORRELL, ${ }^{1}$ AND B. LITT ${ }^{\mathbf{2}}$ \\ ${ }^{1}$ Department of Neurology, Mayo Clinic, Rochester, Minnesota 55905; and ${ }^{2}$ Department of Neurology, \\ Hospital of the University of Pennsylvania, Philadelphia, Pennsylvania 19104
}

This paper was submitted as part of the Special Issue: Understanding Complex Systems

(Complexity, Vol. 10/No. 3 and No. 4)

Received October 14, 2004; revised March 24, 2005; accepted March 24, 2005

\begin{abstract}
Spontaneous energy fluctuations in human hippocampal EEG show prominent amplitude and temporal variability. Here we show hippocampal energy fluctuations often exhibit long-range temporal correlations with power-law scaling. In most cases this scaling behavior persisted on time scales in excess of 10 minutes, the maximum duration we could detect with our recording durations. During these epochs we find that the energy fluctuations exhibit long-range correlations over a broad frequency range $(0.5-100 \mathrm{~Hz})$ with greater persistence of the correlations in the lower frequency bands $(0.5-30 \mathrm{~Hz})$ than the higher $(30-100 \mathrm{~Hz})$. The correlation in hippocampal energy fluctuations is characterized by a bias for energy fluctuations to be followed by similar magnitude fluctuations over all energy scales, i.e. large fluctuations begets large fluctuations and small begets small. (๐ 2005 Wiley Periodicals, Inc. Complexity 10: 35-44, 2005
\end{abstract}

Key Words: EEG; long-range correlations; hippocampus; epilepsy; scaling behavior

\section{INTRODUCTION}

$\mathbf{T}$ he origin and functional correlates of hippocampal EEG oscillations remain active areas of research [1-4]. Hippocampal EEG activity, as measured by depth electrode recordings, is generated by local populations of synchronously firing neurons and when examined over suffi-

Correspondence to: Gregory A. Worrell, E-mail: Worrell.Gregory@mayo.edu ciently long time scales (approximately minutes) exhibits a wide range of amplitude and temporal variation that have received little attention [5]. Unfortunately, most quantitative methods for studying the temporal dynamics of the EEG, such as spectral analysis and nonlinear dynamics [6, 7] require signal stationarity. Although the energy of human hippocampal EEG may remain nearly constant for minutes at a time, the mean energy and variance can also fluctuates widely [8], limiting the usefulness of standard spectral and nonlinear dynamics methods for investigating correlations 
over long time scales. For this reason, complete characterization of the long-range temporal correlations (LRTC) in EEG fluctuations, and in turn the collective neuronal oscillations responsible for the EEG, have received little attention.

In recent years, new methods have been developed that make the problem of analyzing LRTC in nonstationary data tractable. Detrended fluctuation analysis (DFA) is one such method [9-11]. It provides a systematic method for the analysis and characterization of LRTC embedded in nonstationary time series. Detrending the signal on multiple time scales to eliminate spurious detection of temporal correlations that arise as an artifact of signal nonstationarity allows the determination of intrinsic scaling exponents that characterize temporal correlations in mono-fractal signals [12]. Recently, DFA has been used to demonstrate the presence of LRTC with power-law scaling in human EEG recorded from the scalp $[13,14]$.

In this article we analyze a transform of the EEG, which we refer to as the signal energy. This transform is not simply an amplitude measure as it is rarely attains zero values. We use DFA to analyze these energy fluctuations in long continuous EEG time series obtained from intracranial hippocampal depth electrodes placed in five patients with unilateral mesial (amygdalohippocampal) temporal lobe epilepsy undergoing pre-surgical evaluation.

\section{MATERIALS AND METHODS}

\section{EEG Recording}

Intracranial EEG was obtained from chronically implanted depth electrodes in the long axis of the hippocampus of both temporal lobes in five adult epileptic patients being evaluated for epilepsy surgery. The data were recorded using a referential montage and then band-pass analog filtered between 0.1 and $100 \mathrm{~Hz}$ and digitized at $200 \mathrm{~Hz}$.

Five randomly selected 20-minute EEG segments were selected for each patient in each of three states: awake, asleep, and preseizure awake, yielding a total of fifteen 20-minute segments of intracranial EEG for each of the five patients. Seizure onset lead and time were determined by visual inspection by an epileptologist (G.A.W. or B.L.). A second lead was identified, herein referred to as the remote lead, which was in the contralateral hippocampus and electrographically removed from the seizure onset zone.

\section{Determination of Behavioral State}

Continuous video of the patient's entire monitoring stay were viewed to determine the patient's behavioral state (sleep/wake) and identify clinical seizures. Behavior clearly recognized as wake or sleep were recorded, and then the intracranial EEG was quantitatively analyzed to achieve finer resolution of sleep-wake cycles into slow-wave sleep, wakefulness, and indeterminate periods.

\section{Wavelet Filtering}

The data were analyzed in four forms: unfiltered (other than by the analog filter), low-frequency $(0.5-30 \mathrm{~Hz})$, high-frequency $(30-100 \mathrm{~Hz})$, and the standard clinical EEG bands, $\delta$ (0.5-4 Hz), $\theta(4-8 \mathrm{~Hz}), \alpha(8-12 \mathrm{~Hz}), \beta(12-30)$, and $\gamma(30-70$ $\mathrm{Hz}$ ). The offline filtering was performed with a wavelet filtering scheme. Wavelet filtering was selected because of its robust detection of temporally limited frequencies common in EEG tracings.

To facilitate arbitrary frequency band selection, the continuous wavelet transform (CWT) was used despite its significant computational burden relative to the discrete transform (DWT). The CWT computes the wavelet transform at all possible scales, determined by the data set's resolution, within a selected scaling range [15]. The DWT computes the transforms at dyadically selected scales (powers of 2) only. The wavelet transform, $W$, of a one-dimensional signal, $f(x)$, is defined as

$$
W(a, b)=\frac{1}{\sqrt{a}} \int_{-\infty}^{+\infty} f(x) \psi *\left(\frac{x-b}{a}\right) d x
$$

where $a$ represents the wavelet scale, $b$ the wavelet center offset, and $\psi^{*}(x)$ the complex conjugate of the wavelet function. The "gaus4" wavelet, the normalized 4th derivative of a Gaussian, was used because of its compatibility with the CWT, the absence of an imaginary component for computational efficiency, and its relative simplicity.

The scales of a CWT are inversely proportional to the frequencies they best represent. For this reason the number and distribution of scales representing a particular frequency band varies with the band of interest. Fewer scales are devoted to higher frequencies and vice versa. Our filtering function dealt with this by weighting each scale in the CWT by the proportion of the frequency band it represented. The filtered data was generated by applying this weighted average to the CWT coefficients at each time point. The final one-dimensional time series is normalized in magnitude by scaling it by the first-order coefficient generated by a linear regression fit between it and the unfiltered data.

\section{Energy Calculation}

The filtered and unfiltered tracings were transformed to instantaneous energy. The following method was used: First the series of local minima were collected into one time series, and the local maxima into another. From these the 


\section{FIGURE 1}

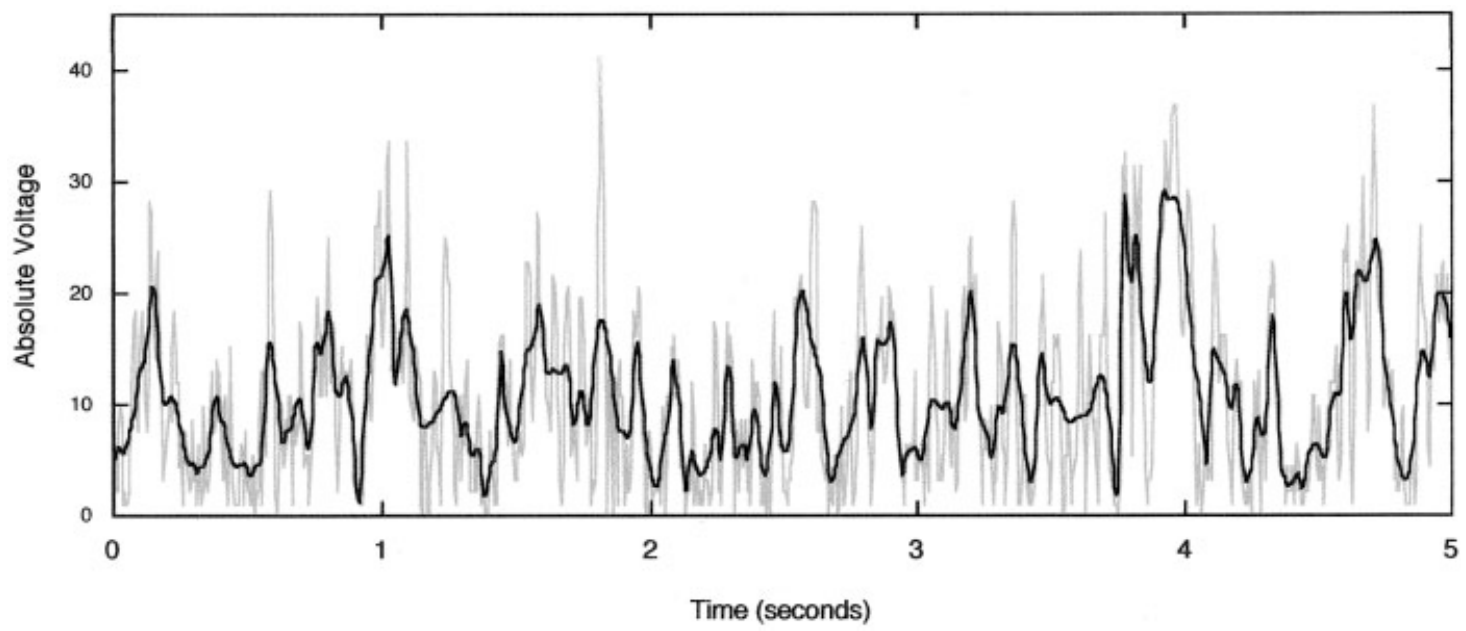

Plot of the absolute value of the EEG voltage (gray) and the energy function (black). The energy function was generated by integrating the data between successive peaks and separately between successive troughs. The integral values were normalized by the length of their respective waveforms and aligned to the original time sequence at the waveform midpoints form a series of anchors. Values between the successive anchor points were generated by cubic spline interpolation.

voltages were integrated between successive maxima and separately between successive minima. The integral values were normalized by their segment lengths. The normalized integrated values were interleaved at their respective waveform midpoints and a cubic spline was used to interpolate between the points (Figure 1). Simply determin- ing the energy from the square of the voltage averaged over a fixed window also produces a self-similar energy time series [5], but these series contains frequent nearzero values corresponding to the zero-crossings in the original data, a characteristic we sought to avoid with this method.

\section{FIGURE 2}
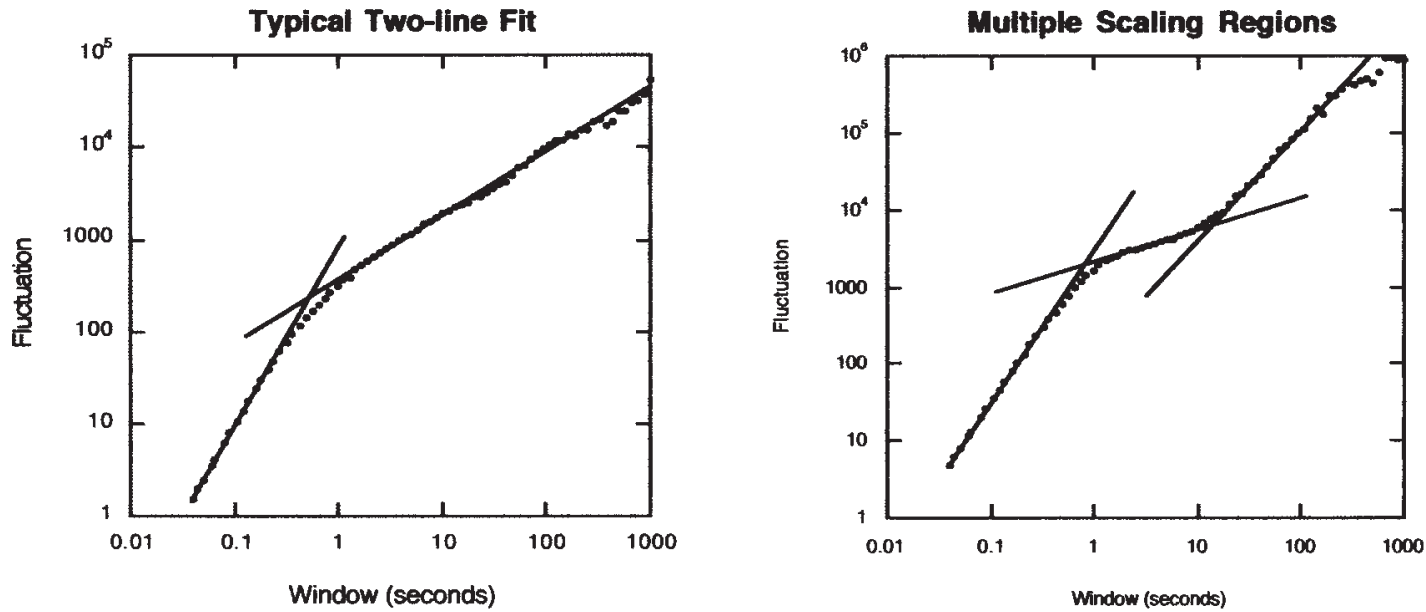

Most of our data were fit with two lines similar to the plot on the left. The lines were chosen by fitting an estimate of the derivative of the DFA function by three line segments: two with zero slope, connected by one of arbitrary slope. The combination of lines that minimized the total deviation was used to select the boundaries for fitting the two lines in the original data. A weighted least squares was used for the final fits. The weighting function was the square root of the number of data windows used to generate each point. Twenty-one of our 450 DFAs were clearly not well represented by a two line fit. These were censored from further analysis. One of these plots, with putative fitting lines is displayed in the right of the figure. 


\section{FIGURE 3}

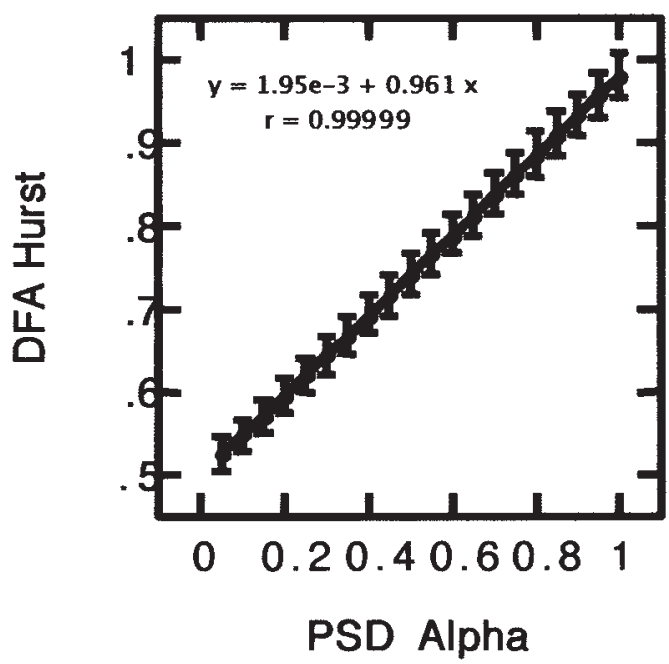

Results of a Monte Carlo simulation using our DFA algorithm. Each point is the mean of the scaling constants derived from $10^{4}$ randomly generated stationary data sets with predesignated scaling constants, each set containing $10^{6}$ points. The scaling constant was derived from the power spectrum (PSD) and DFA for each set. The error bars represent two standard deviations of the DFA scaling constants at each point. One standard deviation was approximately 0.02 at all the points.

\section{Long-range Temporal Coprelations and Detrended Fluctuation Analysis}

For a discrete equidistant voltage time series $\left\{V_{k}\right\}$ the autocorrelation function is

$$
C(s)=\left\langle V_{k} V_{k+s}\right\rangle=\frac{1}{(N-s)} \sum_{k=1}^{N-s} V_{k} V_{k+s}
$$

where $s$ is the time difference between two voltage measurements and $\langle\ldots\rangle$ denotes the ensemble average. For stationary time series spectral analysis, i.e., direct determination of the spectral power density, is often adequate for investigating the autocorrelation function. Long-range temporal correlations characterized by a power-law, $C(s) \approx \mathrm{s}^{-\beta}$, have received considerable attention and recently have been investigated in human EEG recordings [14, 16]. Noisy biological data containing nonstationarities, such as trends, over timescales that are not known a priori make the detection of long duration temporal correlations problematic, and the direct calculation of $C(s)$ or the spectral density is not appropriate. DFA provides an indirect method for characterizing the LRTC in the autocorrelation function and has been found to be a robust estimator of LRTC for noisy biological data containing nonstationary trends $[10,17,18]$.

In DFA the original time series, $\left\{V_{k}\right\}_{N}$, of length $N$ is divided into $N_{l}=N / l$ nonoverlapping windows (segments)

\section{FIGURE 4}

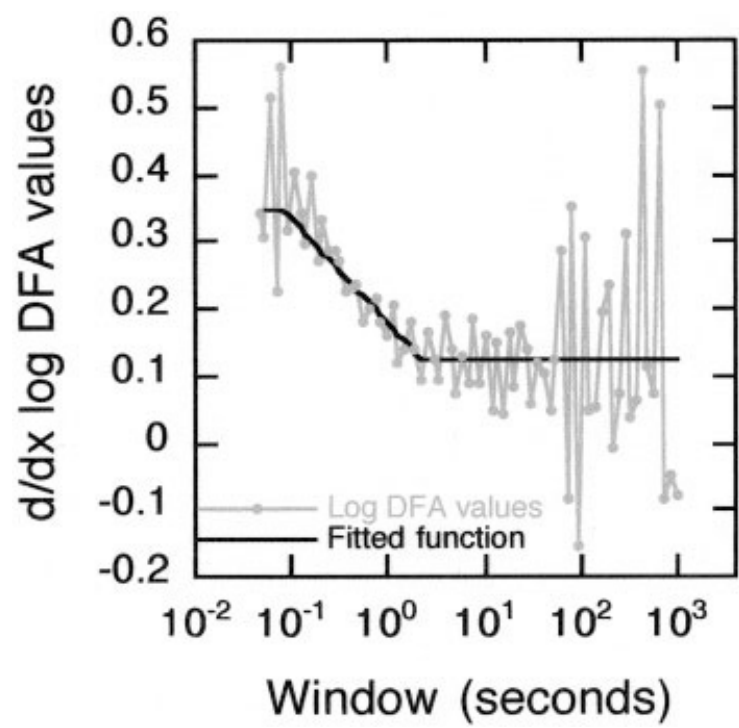

The sets of points selected to for the fits demonstrated in Figure 2 were chosen according to a scheme depicted above. The three line segments, constrained to be zero slope for the first and third, connected by the second, which best fit the derivative of the of the DFA values were calculated. The points encompassed by the first and third segments were fit in the DFA. The intervening points, corresponding to the second line segment were excluded from the fitting subsets.

of length $l$. The correlation structure is studied using the integrated displacement of the original time series $Y_{k}=$ $\sum_{i=1}^{k}\left(V_{i}-\langle V\rangle\right)$, where the mean voltage $\langle V\rangle=1 / N \Sigma_{i=1}^{N} V_{i}$ has been subtracted. Next the detrended integrated time series for each window (or segment) is calculated from $Y_{k}^{l}=Y_{k}-$ $y_{k}^{\text {Trend }}$, where $y_{k}^{\text {Trend }}$ is the local trend in the particular window of calculation (windows of size $l$ ). The variance of the detrended integrated time series in a window $v$ at a particular scale $l$ is

$$
F_{l}^{2}(\nu)=\left\langle\left(Y_{k}^{l}(\nu)\right)^{2}\right\rangle=\frac{1}{l} \sum_{i=1}^{l}\left(Y_{(\nu-1) l+i}^{l}\right)^{2}
$$

The root-mean-square (RMS) fluctuation of the entire detrended time series at the scale $l$ is then obtained by summing over all the individual windows,

$$
F(l)=\sqrt{\frac{1}{N_{l}} \sum_{\nu=1}^{N_{l}} F_{l}^{2}(\nu)}
$$

Long-range temporal correlations (LRTC) are considered present when the autocorrelation function exhibits a power-law decay, $C(s) \approx \mathrm{s}^{-\beta}$, with the exponent character- 
High Freq
Loh Freq

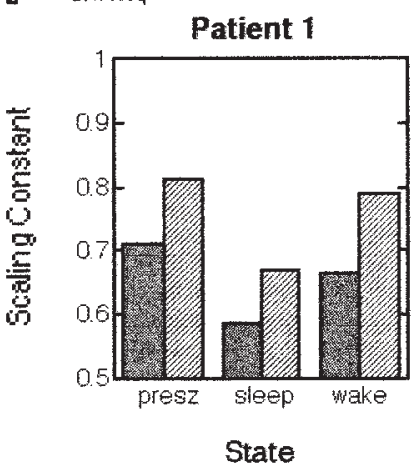

E Hogh Freg

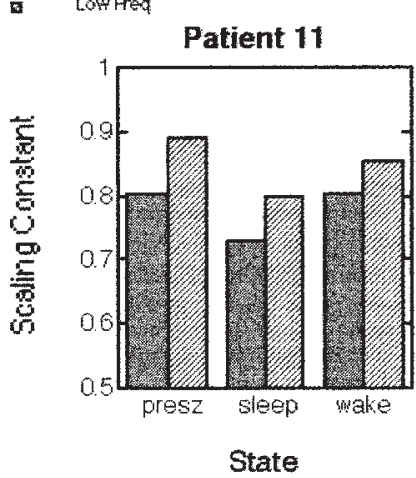

ฮ How Frea

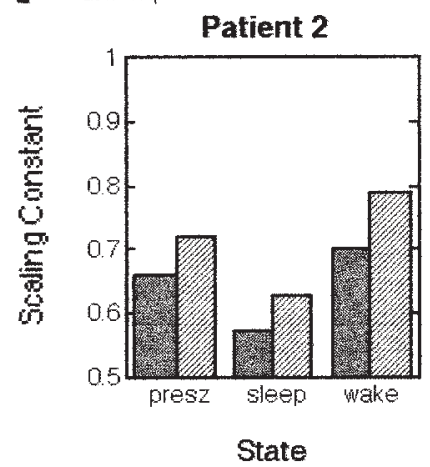

- High Frea

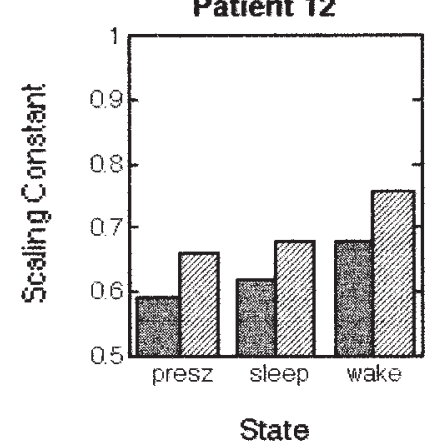

- Hoh Fed

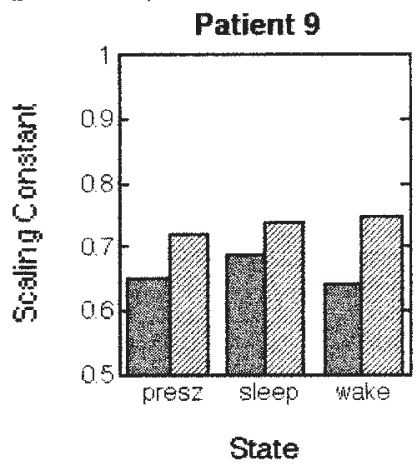

- High Freo

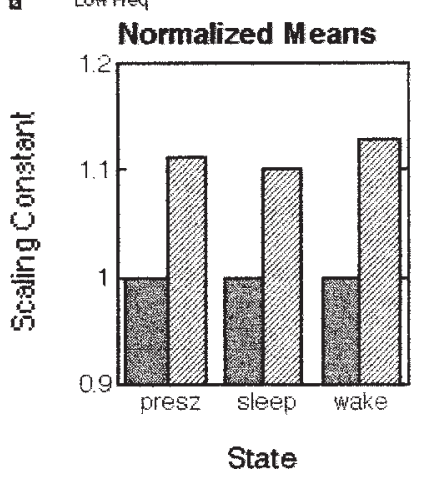

The long-range temporal correlations from each patient in each state were less persistent in the high-frequency range (30-100 $\mathrm{Hz}$ ) than the low range $(0.5-30 \mathrm{~Hz})$. Long-range temporal correlations are present in all conditions. The above data are generated from the seizure onset electrode but are not qualitatively or statistically different in the remote lead. The bottom right graph is generated by normalizing each low-frequency scaling constant by its high-frequency counterpart and averaging across patients to demonstrate the relative average difference in magnitude of the scaling constants across all the patients. Note that error bars are left off this figure as the $t$-tests employed were paired for patient and state. The overall results are statistically significant and the $P$ values are presented in Results.

izing the decay bounded by the relation $0<\beta<1[19,20]$. For the range $0<\beta<1$ the autocorrelation function decays sufficiently slowly such that $\sum_{s=1}^{N} C(s) \approx \mathrm{s}^{1-\beta}$ does not converge for long time scales $(N \rightarrow \infty)$. When the autocorrelation function is characterized by power-law behavior $C(s) \approx \mathrm{s}^{-\beta}$, the RMS fluctuation function $F(l)$ can similarly be shown to exhibit scaling behavior with a related scaling exponent, $F(l) \approx l^{1-\beta / 2}[10,18,21]$.

The scaling exponent characterizing the behavior of the detrended RMS fluctuation function $F(l)$ can be obtained from a $\log$-log plot, i.e., $\log (F(l)) \propto H \log (l)$, where the scaling exponent $H=1-\beta / 2$ is obtained from the slope of the log-log plot by linear regression analysis (Figure 2). The exponent $H$ is often called the Hurst exponent after the famous hydrologist who first identified LRTC in the Nile river levels $[10,12,22]$. The scaling exponent provides a quantitative measure of the intensity of the temporal correlations in the time series $[10,17,20]$. When the voltage fluctuations are asymptotically uncorrelated the asymptotic scaling exponent yields $\beta=1$, or equivalently $(H=1 / 2)$. In this case the correlation function, $C(s) \approx \mathrm{s}^{-1}$, decay sufficiently rapidly that for long time scales the voltage fluctuations become uncorrelated [12]. However, for $0<\beta<1$, or equivalently ( $1>H>1 / 2)$, the decay is sufficiently slow that the correlations persist over long time scales. When $\beta=0$, or $(H=1)$, the autocorrelation function is independent of time, or infinite range, without any decay in the autocorrelation function. Thus, as the scaling exponent $H$ increases from $H=1 / 2 \rightarrow 1(\beta=1 \rightarrow 0)$, the intensity of the correlations increase, and the correlations become more persistent (the autocorrelation function decays less rapidly).

Here the performance of the DFA algorithm was confirmed with simulated time series with specific LRTC characteristics, i.e., particular values of $H$ are easily generated $[12,20]$ and can serve as a test of the analysis method and help to elucidate the manifestations of different scaling 


\section{FIGURE 6}

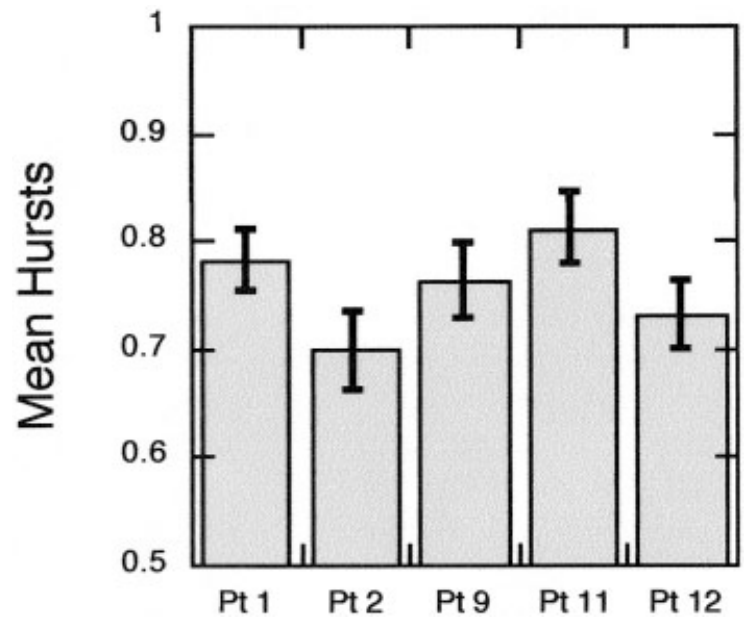

Data generated from the unnormalized Hurst exponents. Included all states (wake, sleep, and preseizure) and leads (onset and remote). Means and errors based on unfiltered energy time series scaling constants.

behavior (Figure 3). The DFA method was checked with simulated data with imposed scaling constants. The artificial data were stationary allowing spectral methods to be used as a reference. Ten thousand novel data sets of $10^{6}$ points each were generated for each scaling constant. The DFA was calculated for each set and the standard deviation of the scaling constants from the collection of the $10^{4}$ sets. The spectral scaling constant was also derived for each set as the negative slope of the fit a $\log$ frequency versus log power plot.

In contrast to the simulated data with a single scaling constant, most of the human EEG DFA curves had two distinct regions: a linear region at short time scales that was steeper with an exponent outside the range characteristic of LRTC scaling and a second linear region at longer time scales. We developed a systematic method to fit the two regions. The derivative of the DFA curve was computed, and three lines were fit to the derivative with two of zero-slope at either ends of the DFA curve, connected by a segment of arbitrary slope (Figure 4). The set of three lines which best fit the data determined the cut points for fitting the two lines to the original DFA points. Thus the exact temporal limits of the fits are determined algorithmically for each DFA tracing and are not identical between tracings. In general the time range for fitting the second scaling region corresponded to the period of the dominant frequency (e.g., 1 second for delta band oscillations) at the low end to then end of our ability to detect scaling $(\sim 10$ minutes in these 20-minute EEG segments). The reasoning belying fitting the short time scale regions of the DFA curves is twofold: to point out the existence of this region and to determine the range of points to be fit in the longer range scaling regions.

\section{Statistics}

Five 20-minute segments of EEG were analyzed for each condition (Figure 5). The variables were patient, behavioral state, and frequency band, and recording lead. A significant patient effect was demonstrated (Figure 6). The magnitude of the patient effect was great enough to obscure from statistical significance many of the effects investigated in this study. To work around this, in some further analyses each scaling constant was normalized by subtracting the patient mean obtained from all states, bands, and leads and adding the grand mean obtained from all states, bands, leads, and patients. These normalized scaling constants were then subjected to two-tailed $t$-tests in various pairings as described in Results.

\section{RESULTS}

The energy transforms of the intracranial EEG displayed power law scaling by DFA analysis in 429 of the 450 data sets analyzed. Twenty-one data sets were excluded from statistical analysis for DFA plots that appeared to contain more than two linear regions such as seen in Figure 2. Every DFA plot contained at least two linear regions, the first and steepest region being a consequence of the dominant frequency in the tracing, which in the case of the lowest frequencies can be an artifact introduced by filtering. The scaling constants in this region were uniformly greater than one, beyond the range defined for LRTCs. The scaling constants analyzed in this article were those derived from the

\section{FIGURE 7}

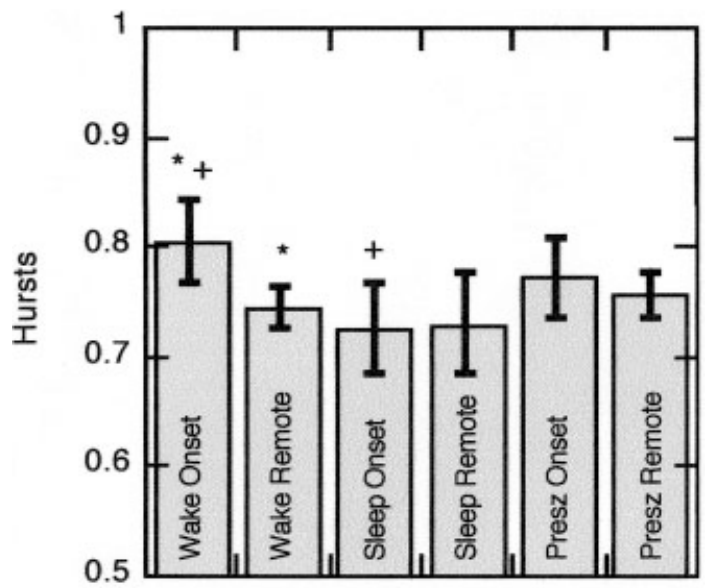

State

Data generated from the patient-normalized Hurst exponents. Means and errors based on unfiltered energy time series scaling constants. 


\section{FIGURE 8}

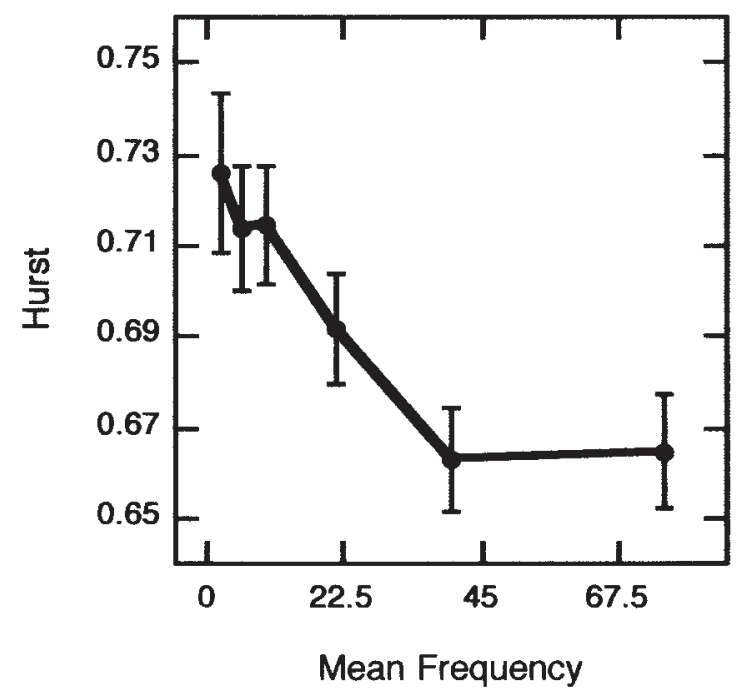

Data generated from the patient normalized Hurst exponents. Included all states (wake, sleep, and preseizure) and leads (onset and remote).

second linear region of the plots, of the included 429 analyses the scaling constants all fell between 0.5 and 1.0, the range defining long-range scaling behavior.

Averages of the scaling constants under each behavioral state, lead, and frequency band were computed so that paired $t$-tests could be performed. Under this method of analysis few of our comparisons met statistic significance, likely due to undersampling. However, when the scaling constant means paired for patient and behavioral state were compared between coarsely divided low- and high-frequency bands, defined as $0.5-30 \mathrm{~Hz}$, and $30-100 \mathrm{~Hz}$, respectively, the low-frequency filtered data showed significantly larger scaling constants than the higher frequency bands in both the seizure onset and remote leads $\left(\mathrm{p}=1.3 \times 10^{-9}\right.$ onset, $\mathrm{p}=3.6 \times 10^{-5}$ remote). The results for the seizure onset lead are depicted in Figure 5, separated by patient to demonstrate the consistency of the finding; a normalized plot demonstrates the relatively equivalent magnitudes of the scaling differences across states.

Normalized scaling constants were generated on the energy transforms of unfiltered EEG and compared as shown in Figure 7. A significant difference was noted between the onset and remote leads during wakefulness, this result replicates the result of a more comprehensive study of this question published previously [16]. Also noted in this figure is that the scaling appears to be greater during wakefulness than sleep in the seizure onset leads.

Using the patient-normalized scaling constants the frequency relationship demonstrated in Figure 8 was found. The data in this figure were the scaling constants in all behavioral states. The grid points represent the centers of the classical clinical EEG bands defined here as $\delta(0.5-4 \mathrm{~Hz})$, $\theta(4-8 \mathrm{~Hz}), \alpha(8-12 \mathrm{~Hz}), \beta(12-30 \mathrm{~Hz})$, and $\gamma(30-50 \mathrm{~Hz})$. We further analyzed the remainder of the spectrum as a single band defined as "high frequency." (hf: $50-100 \mathrm{~Hz}$ ). There is a clear decline in the magnitude of the scaling of the energy function as a function of frequency in the clinical EEG

\section{FIGURE 9}
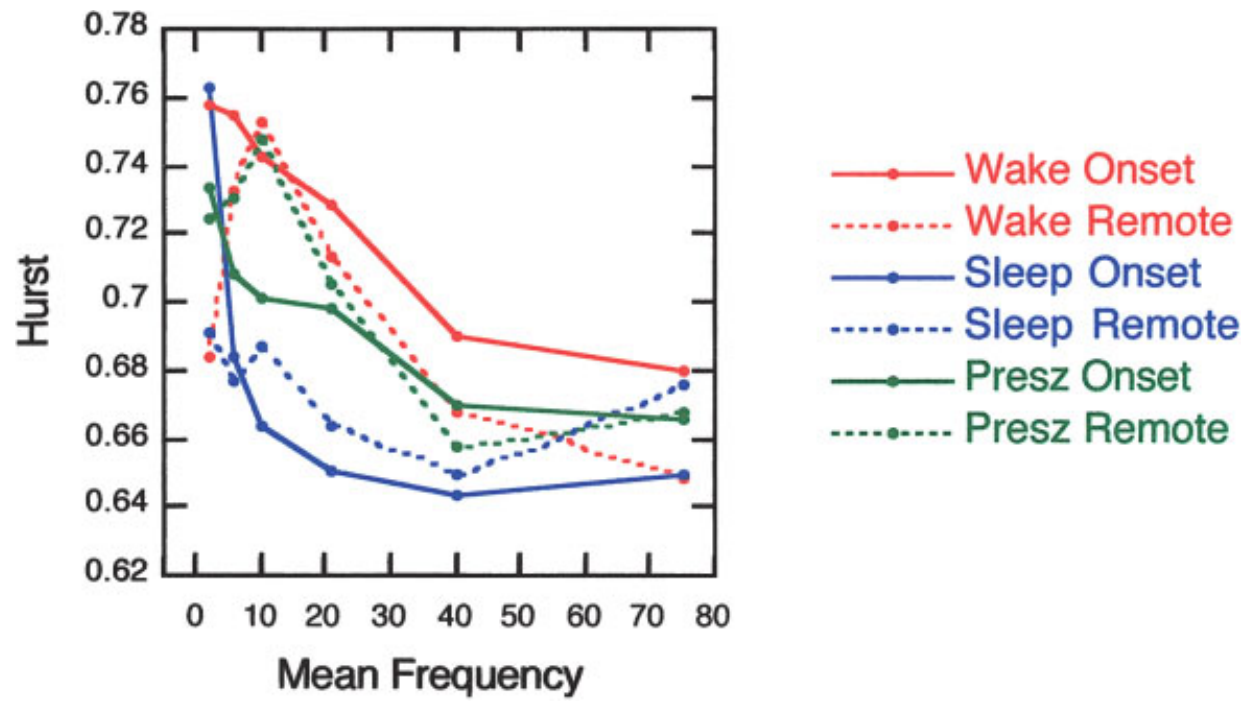

Scaling exponents plotted as a function of frequency. Error bars are excluded for clarity but the results of $t$-tests comparing various frequency bands and states are shown in Tables 1 and 2. 


\section{TABLE 1}

$P$ Values of Two-Tailed Unpaired $t$-Tests of the Patient-Normalized Scaling Exponents of the Energy Time Series

\begin{tabular}{lcc}
\hline Presz vs. sleep & $p_{\text {onset }}$ & $p_{\text {remote }}$ \\
\hline$\delta$ & $4.12 \mathrm{E}-01$ & $1.95 \mathrm{E}-01$ \\
$\theta$ & $3.65 \mathrm{E}-01$ & $\mathbf{2 . 5 3 E - 0 2}$ \\
$\alpha$ & $1.02 \mathrm{E}-01$ & $\mathbf{1 . 2 6 \mathrm { E } - 0 2}$ \\
$\beta$ & $5.42 \mathrm{E}-02$ & $\mathbf{3 . 6 7 \mathrm { E } - 0 2}$ \\
$\gamma$ & $3.27 \mathrm{E}-01$ & $6.01 \mathrm{E}-01$ \\
$\mathrm{hf}$ & $6.35 \mathrm{E}-01$ & $6.33 \mathrm{E}-01$ \\
\hline
\end{tabular}

\begin{tabular}{lcc} 
Presz vs. wake & $\mathrm{p}_{\text {onset }}$ & $\mathrm{p}_{\text {remote }}$ \\
\hline$\delta$ & $4.80 \mathrm{E}-01$ & $6.34 \mathrm{E}-02$ \\
$\theta$ & $5.66 \mathrm{E}-02$ & $9.06 \mathrm{E}-01$ \\
$\alpha$ & $5.04 \mathrm{E}-02$ & $7.92 \mathrm{E}-01$ \\
$\beta$ & $1.19 \mathrm{E}-01$ & $6.01 \mathrm{E}-01$ \\
$\gamma$ & $3.28 \mathrm{E}-01$ & $4.80 \mathrm{E}-01$ \\
hf & $4.79 \mathrm{E}-01$ & $2.64 \mathrm{E}-01$ \\
\hline
\end{tabular}

\begin{tabular}{lcc} 
Sleep vs. wake & $p_{\text {onset }}$ & $p_{\text {remote }}$ \\
\hline$\delta$ & $8.85 \mathrm{E}-01$ & $7.98 \mathrm{E}-01$ \\
$\theta$ & $\mathbf{3 . 9 7 \mathrm { E } - 0 3}$ & $\mathbf{2 . 2 6 \mathrm { E } - 0 2}$ \\
$\alpha$ & $\mathbf{2 . 3 6 \mathrm { E } - 0 4}$ & $\mathbf{3 . 1 3 \mathrm { E } - 0 3}$ \\
$\beta$ & $\mathbf{1 . 2 1 \mathrm { E } - 0 3}$ & $\mathbf{4 . 9 9 \mathrm { E } - 0 3}$ \\
$\gamma$ & $8.32 \mathrm{E}-02$ & $1.75 \mathrm{E}-01$ \\
hf & $3.14 \mathrm{E}-01$ & $1.03 \mathrm{E}-01$
\end{tabular}

Values compare states separated by frequency and lead. Results significant at the 0.05 level are boldface type.

bands. This trend is seen in the unnormalized data (Figure 5), but the patient-normalized scaling constants allow for detection of statistical differences between the more finely defined EEG bands.

Figure 9 shows the frequency dependence of the patientnormalized scaling constants divided by behavioral state and lead. Error bars are left off this plot for clarity but the results of the two-tailed tests comparing the various variables are presented in Tables 1 and 2. These tests were performed on the patient-normalized scaling exponents. Table 1 shows the $P$ values of comparisons between states for each frequency band and lead. Table 2 shows the $P$ values of comparisons between frequency bands for each state and lead. In summary, we detected significant differences in scaling between sleep and wakefulness in the 4- to $30-\mathrm{Hz}$ range on both the onset and remote leads. In the comparison of preseizure (which was defined to arise out of wakefulness) versus sleep this difference persisted in the remote leads but was lost in the onset leads. No differences in scaling were noted between the preseizure and awake state in any of the bands. Table 2 demonstrates a significant difference between the low- $(4-30 \mathrm{~Hz})$ and high-frequency (30-100 Hz) band scaling constants in the wake state. This difference is also seen in the preseizure state in the remote lead, but is lost in the onset lead. The difference is not seen in sleep, where instead the onset lead there is a clear difference between delta and all of the higher frequency bands, but in the remote lead no significant differences exist between any of the bands.

\section{DISCUSSION}

We have demonstrated that for long epochs (minutes) the energy fluctuations in human hippocampal EEG exhibit power-law scaling and LRTC. The scaling exponent provides a quantitative measure of the temporal correlations that exist in the energy time series. When the signal is completely uncorrelated (Gaussian or non-Gaussian probability distributions), the calculation of the scaling exponent yields $H=0.5$. This is easily demonstrated by randomly shuffling the energy time series. When applied to EEG signal we found LRTC and power-law scaling with scaling exponents $0.5<H<1$. When $0.5<H \leq 1$, the data are correlated such that large (small) energy fluctuations are likely to be followed by large (small) energy fluctuations, i.e., clustering of extreme energy fluctuations. When $H=1$ the LRTC become independent of time with infinite range, and as $H$ increases from $H \geq 0.5$ toward $H=1$, the temporal correlations in the time series are more persistent (decay more slowly with time).

The physiologic mechanism(s) responsible for LRTC of hippocampal energy fluctuations energy fluctuations are not known, but simulations demonstrate [17] that the introduction of a biased probability distributions can produce LRTC. Biased probability distributions differ in the frequency of occurrence of their extreme values from the expected frequencies in random models such as Gaussian or Poisson distributions. Although LRTC in the temporal dynamics do not implicate a unique mechanism, recent studies show that aggregate time series made up of elements with heavy-tailed distributions (i.e., biased toward the occurrence of extreme values) exhibit similar behavior [23].

We further found that there appears to be a patient dependent effect on the scaling constants of the energy time series. It is not clear from our data set whether this phenomenon stems from a meaningful difference in the EEG of different patients or whether it may be an artifact of small differences in the recording configurations. The latter is suggested as electronic noise displays scaling behavior independent of biological signal. As the origin of this patient difference is unknown at this time we have refrained from drawing conclusions from the patient-normalized data, though we believe them to be meaningful, and they in general corroborate the unnormalized conclusions. 


\section{TABLE 2}

$P$ Values of Two-Tailed Unpaired $t$-Tests of the Patient-Normalized Scaling Exponents of the Energy Time Series

\begin{tabular}{|c|c|c|c|c|c|}
\hline Presz onset & $\theta$ & $\alpha$ & $\beta$ & $\gamma$ & hf \\
\hline$\delta$ & 4.15E-01 & 2.81E-01 & $2.25 \mathrm{E}-01$ & $3.46 \mathrm{E}-02$ & 3.03E-02 \\
\hline$\theta$ & & $7.90 \mathrm{E}-01$ & 6.88E-01 & $1.26 \mathrm{E}-01$ & $9.98 \mathrm{E}-02$ \\
\hline$\alpha$ & & & 8.93E-01 & $1.70 \mathrm{E}-01$ & 1.29E-01 \\
\hline$\beta$ & & & & $1.88 \mathrm{E}-01$ & $1.40 \mathrm{E}-01$ \\
\hline$\gamma$ & & & & & 8.30E-01 \\
\hline
\end{tabular}

\begin{tabular}{|c|c|c|c|c|c|}
\hline Presz remote & $\theta$ & $\alpha$ & $\beta$ & $\gamma$ & hf \\
\hline$\delta$ & 7.17E-01 & $2.38 \mathrm{E}-01$ & $2.85 \mathrm{E}-01$ & 1.43E-04 & 1.76E-03 \\
\hline$\theta$ & & 4.17E-01 & $1.85 \mathrm{E}-01$ & 1.41E-04 & $1.48 \mathrm{E}-03$ \\
\hline$\alpha$ & & & 4.60E-02 & 3.20E-05 & 3.46E-04 \\
\hline$\beta$ & & & & $9.62 \mathrm{E}-03$ & 4.89E-02 \\
\hline$\gamma$ & & & & & 5.70E-01 \\
\hline
\end{tabular}

\begin{tabular}{|c|c|c|c|c|c|}
\hline Sleep onset & $\theta$ & $\alpha$ & $\beta$ & $\gamma$ & hf \\
\hline$\delta$ & 1.33E-02 & $1.20 \mathrm{E}-03$ & 6.22E-04 & 8.47E-04 & $5.90 \mathrm{E}-03$ \\
\hline$\theta$ & & 4.07E-01 & $2.15 \mathrm{E}-01$ & $1.71 \mathrm{E}-01$ & $3.21 \mathrm{E}-01$ \\
\hline$\alpha$ & & & $5.95 \mathrm{E}-01$ & 4.54E-01 & $6.58 \mathrm{E}-01$ \\
\hline$\beta$ & & & & 8.06E-01 & $9.76 \mathrm{E}-01$ \\
\hline$\gamma$ & & & & & $8.71 \mathrm{E}-01$ \\
\hline
\end{tabular}

\begin{tabular}{|c|c|c|c|c|c|}
\hline Sleep remote & $\theta$ & $\alpha$ & $\beta$ & $\gamma$ & hf \\
\hline$\delta$ & $6.21 \mathrm{E}-01$ & 8.76E-01 & $2.81 \mathrm{E}-01$ & $9.79 \mathrm{E}-02$ & 5.92E-01 \\
\hline$\theta$ & & $6.99 \mathrm{E}-01$ & 5.61E-01 & 2.16E-01 & $9.68 \mathrm{E}-01$ \\
\hline$\alpha$ & & & 2.94E-01 & 7.51E-02 & $6.45 \mathrm{E}-01$ \\
\hline$\beta$ & & & & $4.40 \mathrm{E}-01$ & 5.31E-01 \\
\hline$\gamma$ & & & & & $9.48 \mathrm{E}-02$ \\
\hline
\end{tabular}

\begin{tabular}{|c|c|c|c|c|c|}
\hline Wake onset & $\theta$ & $\alpha$ & $\beta$ & $\gamma$ & hf \\
\hline$\delta$ & $8.82 \mathrm{E}-01$ & $5.58 \mathrm{E}-01$ & $2.54 \mathrm{E}-01$ & $1.56 \mathrm{E}-02$ & 4.44E-03 \\
\hline$\theta$ & & $5.48 \mathrm{E}-01$ & 1.63E-01 & $2.40 \mathrm{E}-03$ & 4.57E-04 \\
\hline$\alpha$ & & & 3.99E-01 & $7.62 E-03$ & $1.62 \mathrm{E}-03$ \\
\hline$\beta$ & & & & 4.35E-02 & 1.17E-02 \\
\hline$\gamma$ & & & & & $6.19 \mathrm{E}-01$ \\
\hline
\end{tabular}

\begin{tabular}{|c|c|c|c|c|c|}
\hline Wake remote & $\theta$ & $\alpha$ & $\beta$ & $\gamma$ & hf \\
\hline$\delta$ & 3.66E-02 & 2.21E-03 & $1.42 \mathrm{E}-01$ & 4.34E-01 & 8.75E-02 \\
\hline$\theta$ & & $2.89 \mathrm{E}-01$ & $2.59 \mathrm{E}-01$ & 4.55E-04 & $1.42 \mathrm{E}-05$ \\
\hline$\alpha$ & & & $1.01 \mathrm{E}-02$ & $9.50 \mathrm{E}-07$ & 2.07E-08 \\
\hline$\beta$ & & & & 8.53E-04 & 2.18E-05 \\
\hline$\gamma$ & & & & & $1.80 \mathrm{E}-01$ \\
\hline
\end{tabular}

Values compare scaling between frequencies, separated by state and lead. Results significant at the 0.05 level are boldface type. 


\section{CONCLUSION}

In this article we show that broad-band $(0.5-100 \mathrm{~Hz})$ energy fluctuations in human hippocampus exhibit LRTC with power-law scaling. The correlations in energy fluctuations are more persistent at low frequency $(0.5-30 \mathrm{~Hz})$ than at the higher frequency range. The presence of long-range temporal correlations in the temporal dynamics of energy fluctuations of human hippocampus does not implicate a unique mechanism, and recent studies show that self-similar time series with LRTC can arise from the aggregate time series made up of elements with heavy tailed distribution [23].

\section{ACKNOWLEDGMENTS}

This work was supported by the National Institutes of Health Grants K23 NS047495-01 (GAW), RO1 NS041811-01, and MH-62298RO1 (B.L.).

\section{REFERENCES}

1. Buzsaki, G. Theta oscillations in the hippocampus. Neuron 2002, 33(3), 325-340.

2. Howard, M.; Rizzuto, D.; Caplan, J.; Madsen, J.; Lisman, J.; Aschenbrenner-Scheibe, R.; Schulze-Bonhage, A.; Kahana, M. Gamma oscillations correlate with working memory load in humans. Cerebral Cortex 2003, 13(12), 1369-1374.

3. Kahana, M.; Sekuler, R.; Caplan, J.; Kirschen, M.; Madsn, J. Human theta oscillations exhibit task dependence during virtual maze. Journal Neuroscience, 1999, 399, 781-784.

4. Lisman, J.; Idiart, M. Storage of $7+2$ short-term memories in oscillatory subcycles. Science 1995, 267, 1512-1515.

5. Worrell, G.; Cranstoun, S.D.; Echauz, J.; Litt, B. Evidence for SOC in human epileptic hippocampus. NeuroReport 2002, 13(16), $2017-2021$.

6. Palus, M. Nonlinearity in normal human EEG: Cycles, temporal asymmetry, nonstationarity and randomness, not chaos. Biol Cybern 1996, 75(5), 389-396.

7. Stam, C.J., Pijn, J.P.; Suffczynski, P.; Lopes da Silva, F.H. Dynamics of the human alpha rhythm: Evidence for non-linearity? Clin Neurophysiol 1999, 110(10), 1801-1813.

8. Cranstoun, S.D.; Ombao, H.C.; von Sachs, R.; Guo, W.; Litt, B. Time-frequency spectral estimation of multichannel EEG using the auto-SLEX method. IEEE Trans Bioeng 2002, 49(9), 988-996.

9. Buldyrev, S.V.; Goldberger, A.L.; Havlin, S.; Mantegna, R.N.; Matsa, M.E.; Peng, C.K.; Simons, M.; Stanley, H.E. Long-range correlation properties of coding and noncoding DNA sequences: GenBank analysis. Phys Rev E Stat Phys Plasmas Fluids Relat Interdiscip Topics 1995, 51(5), 5084-5091.

10. Peng, C.-K.; Havlin, S.; Stanley, H.E.; Goldberger, A.L. Quantification of scaling exponents and crossover phenomena in nonstationary heartbeat time series. Chaos 1995, 5, 82-87.

11. Ivanov, P.; Bunde, A.; Amaral, L.A.; Havlin, S.; Fritsch-Yelle, J;. Baevsky, R.M.; Stanley, H.E.; Goldberger, A.L. Sleep-wake differences in scaling behavior of the human heartbeat: analysis of terrestrial and long-term space flight data. Europhys Lett 1999, 48(5), 594-600.

12. Mandelbrot, B. Gaussian Self-Affinity and Fractals; Springer-Verlag: Berlin, 2002.

13. Ferree, T; Hwa, R. Power-law scaling in human EEG: Relation to Fourier power spectrum. Neurocomputing 2003. 52, 755-761.

14. Linkenkaer-Hansen, K.; Nikouline, V.V.; Palva, J.M.; IImoniemi, R.J. Long-range temporal correlations and scaling behavior in human brain oscillations. J Neurosci 2001, 21(4), 1370-1377.

15. Goelz, H.; Jones, R.D.; Bones, P.J. Wavelet analysis of transient biomedical signals and its application to detection of epileptiform activity in the EEG. Clin Electroencephalog 2000, 31(4), 181-191.

16. Parish L.M.; Worrell, G.A.; Cranstoun, S.D.; Stead, M.; Pennell, P.; Litt, B. Long-range temporal correlations in epileptogenic and non-epileptogenic human hippocampus. Neuroscience 2004, 125(4), 1069-1076.

17. Feder, J. Fractals; 1988, Plenum: New York.

18. Kantelhardt, J.W.; Koscielny-Bunde, E.; Rego, H.H.A.; Havlin, S.; Bunde, A. Detecting long-range correlations with detrended fluctuation analysis. 2001, Physica A Stat Mech Appl 295, 441-454.

19. Beran, J. Statistics for Long-Memory Processes; CRC Press; 1994.

20. Rangarajan, G.; Ding, M. Integrated approach to the assessment of long range correlation in time series data. Phys Rev E Stat Phys Plasmas Fluids Relat Interdiscip Topics 2000, 61(2), 4991-5001.

21. Taqqu, M.S.; Teverovsky, V.; Willinger, W. Estimators for long-range dependence: An empirical study. Fractals 1995, 3, 785-798.

22. Hurst, H.E.; Black, R.P.; Simaika, Y.M. Long-term storage. An experimental study. Constable: London, 1965.

23. Willinger, W.; Taqqu, M.; Sherman, R.; Wison, D. Self-similarity through high-variability: statistical analysis of Ethernets LAN traffic at the source level. IEEE/ACM Trans. Networking 1997, 5, 1-15. 